\title{
Photodynamic therapy for polypoidal choroidal vasculopathy secondary to choroidal nevus
}

This article was published in the following Dove Press journal:

International Medical Case Reports Journal

14 February 2017

Number of times this article has been viewed

\author{
James G Wong ${ }^{1-3}$ \\ Xin Jie Lai' \\ Richard Y Sarafian ${ }^{4}$ \\ Hon Seng Wong ${ }^{5}$ \\ Jeremy B Smith
}

'Strathfield Retina Clinic, Sydney, NSW, Australia; ${ }^{2}$ Medical Retina Unit, Sydney Eye Hospital, Sydney, NSW, Australia; ${ }^{3}$ Save Sight Institute, University of Sydney, Sydney, NSW, Australia; ${ }^{4}$ School of Medicine, University of Queensland, Brisbane, QLD, Australia; ${ }^{5}$ Department of Ophthalmology, Universiti Kebangsaan Malaysia Medical Centre, Kuala Lumpur, Malaysia; ${ }^{6}$ Ophthalmology Clinic, Children's Hospital Westmead, Sydney, NSW, Australia
Correspondence: Xin Jie Lai Strathfield Retina Clinic, Suite 3A/9 Redmyre Road, Strathfield, Sydney, NSW 2135, Australia

Tel +6I 297463378

Fax +6I 297463396

Email research@strathfieldretina.com.au

\begin{abstract}
We report a case of a Caucasian female who developed active polypoidal choroidal vasculopathy (PCV) at the edge of a stable choroidal nevus and was successfully treated with verteporfin photodynamic therapy. No active polyp was detectable on indocyanine green angiography 2 years after treatment, and good vision was maintained. Indocyanine green angiography is a useful investigation to diagnose PCV and may be underutilized. Unlike treatment of choroidal neovascularization secondary to choroidal nevus, management of PCV secondary to nevus may not require intravitreal anti-vascular endothelial growth factor therapy. Photodynamic monotherapy may be an effective treatment of secondary PCV.

Keywords: chorioretinopathy, indocyanine green angiography, optical coherence tomography, OCT, fluorescein angiography, pigment epithelium detachments, PEDs, subretinal fluid
\end{abstract}

\section{Introduction}

Choroidal nevus is the most common adult intraocular tumor of the ocular fundus. It is a benign melanocytic lesion typically $<2 \mathrm{~mm}$ in thickness, with an annual malignant transformation rate of one in 8,845. ${ }^{1}$ Polypoidal choroidal vasculopathy (PCV) is a recurrent and relapsing chorioretinopathy characterized by grape-like subretinal vascular lesions associated with retinal pigment epithelium detachments (PEDs). ${ }^{2}$ Indocyanine green angiography (ICGA) is the gold standard for PCV diagnosis. However, patients with PCV are often mistaken for patients with exudative age-related macular degeneration and typical choroidal neovascularization (CNV). PCV associated with choroidal nevus is a combination rarely seen in clinical practice. We previously reported the first case in the literature of a stable PCV associated with nevus, which was managed conservatively. ${ }^{3}$ In this article, we performed a retrospective chart review following the written informed patient consent of a 78-year-old Caucasian female, who had active, symptomatic PCV secondary to nevus, and was successfully treated with photodynamic therapy (PDT). The patient also gave written informed consent to publish this case report.

\section{Case report}

This case involved a 78-year-old Caucasian female with a stable left-eye superotemporal extrafoveal pigmented nevus for 20 years. Her left eye had a sudden onset of paracentral scotoma for 1 week. Best-corrected visual acuity was 6/6 (0.0 logMAR) in each eye. Funduscopy showed a pigmented lesion measuring $4.8 \times 3.2 \mathrm{~mm}$ in basal dimensions with overlying clumped soft drusen at the posterior pole along the $2 \mathrm{o}$ 'clock meridian (Figure 1A), corresponding to a nevus at 2.0 disc diameters from fovea. A 

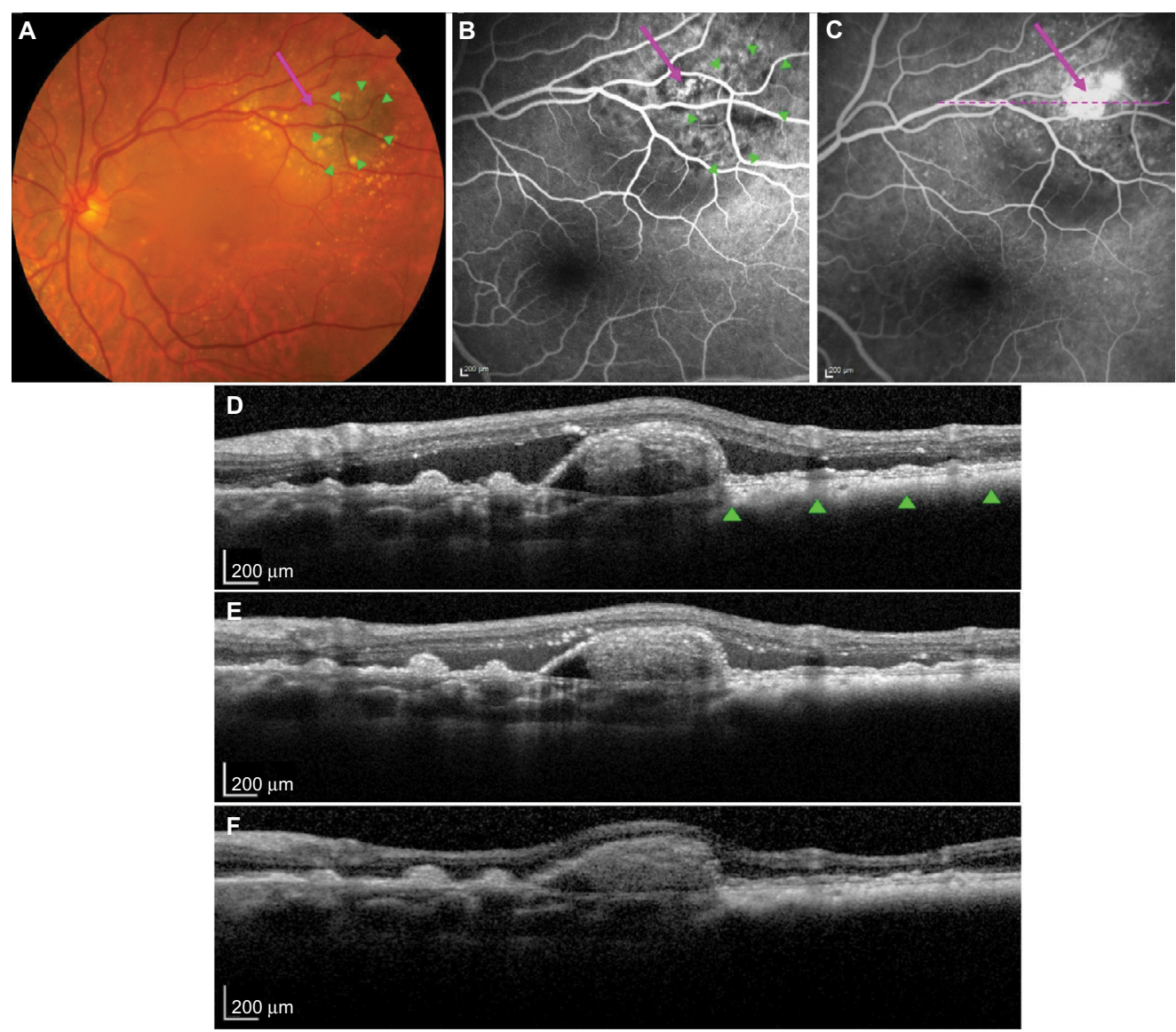

Figure I PCV secondary to a stable choroidal nevus.

Notes: Arrows point to the PCV lesion in (A)-(C). Green triangles outline the nevus lesion in (A), (B), and (D). (A) Color fundus photography shows a pigmented choroidal nevus lesion with overlying clumped soft drusen. (B) Fluorescein angiogram shows a polypoidal lesion at the nasal edge adjacent to the choroidal nevus with early filling of the polypoidal lesion after dye injection (20 seconds). (C) Hyperfluorescence in the late phase ( 3 minutes); dotted line shows the OCT cross-sectional slice. (D) OCT reveals extensive SRF and a retinal PED with underlying polypoidal lesion. Following PDT, OCT shows (E) reduced SRF at 2 weeks and (F) an absence of SRF at 2 months. Abbreviations: PCV, polypoidal choroidal vasculopathy; OCT, optical coherence tomography; SRF, subretinal fluid; PED, pigment epithelium detachment; PDT, photodynamic therapy.

discreet orange nodule adjacent to the pigmented lesion on the nasal aspect and associated subretinal fluid (SRF) was noted. Scattered large soft drusen was observed bilaterally.

Optical coherence tomography (Heidelberg Engineering, Heidelberg, Germany) showed typical features of a flat nevus with PED associated with an underlying discreet polyp-like lesion at the nasal edge of the nevus and extensive SRF (Figure 1D). Fluorescein angiography (Figure 1B and C) and ICGA (Heidelberg Engineering; Figure 2A and B) demonstrated early filling of a grape-like structure suggestive of PCV with leakage in the late phase. Pulsation of the PCV lesion was noticed on ICGA. ICGA also revealed an associated small branching vascular network (BVN) in both early and late phases (Figure 2A and B).

A diagnosis of PCV adjacent to nevus was made. Due to the peripheral location of PCV and the presence of SRF threatening the fovea, PDT was performed after written informed consent was obtained. Intravenous verteporfin $\left(6 \mathrm{mg} / \mathrm{m}^{2}\right)$ was followed by standard-fluence PDT $\left(50 \mathrm{~J} / \mathrm{cm}^{2}\right)$ at the center of the PCV with a spot size of $1,600 \mu \mathrm{m}$ over 83 seconds from a $689 \mathrm{~nm}$ laser. Following treatment, the patient noted an improvement in her paracentral scotoma. SRF was reduced at 2 weeks (Figure 1E). At 2 months, SRF was absent; however, a persisting small PED was observed (Figure 1F). ICGA at 7 months showed an absence of leakage, with complete regression of the polypoidal lesion (Figure $2 \mathrm{C}$ and $\mathrm{D}$ ). No active polyp could be detected on ICGA at 2 years, with a stable best-corrected visual acuity of $6 / 6(0.0 \log \mathrm{MAR})$.

\section{Discussion}

Studies have found that patients with choroidal nevus may develop typical CNV..$^{4-7}$ However, PCV secondary to nevus has rarely been reported. We previously reported a case of a quiescent PCV arising from a stable choroidal nevus where the polyp was located above the nevus, however, between the retinal pigment epithelium (RPE) and Bruch's membrane. This 


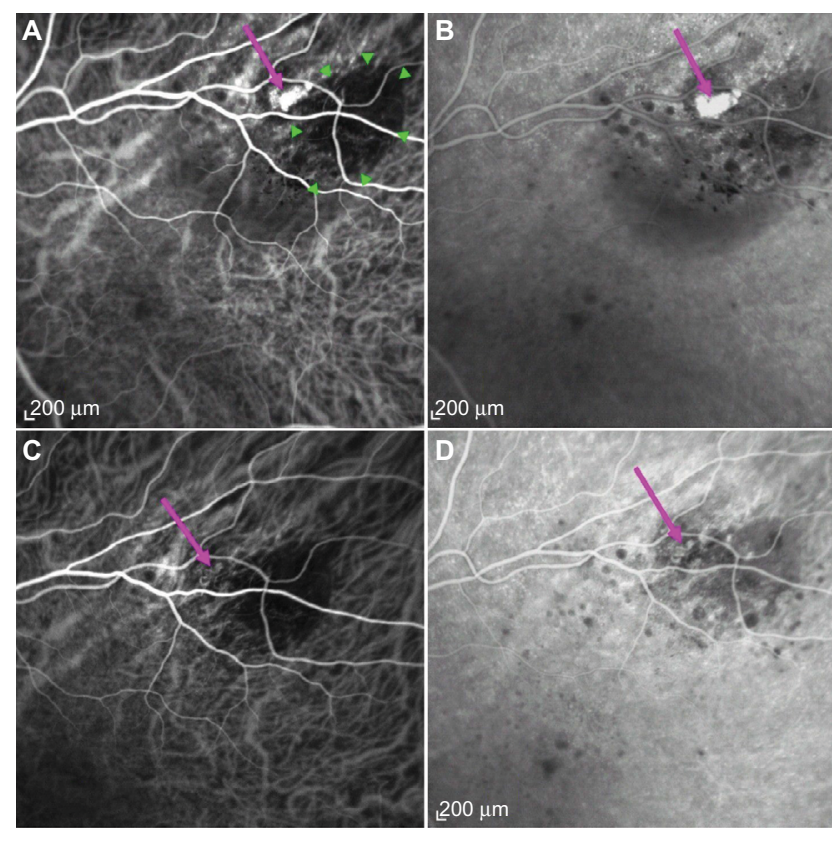

Figure 2 Regression of polyp after PDT laser.

Notes: ICGA shows a choroidal nevus (green triangles in $\mathbf{A}$ ) and a grape-like polypoidal lesion (arrows in A-D) with small BVN located at the nasal edge adjacent to the nevus lesion. (A) Hypercyanescence of the polypoidal lesion was observed in ICGA in the early phase ( 30 seconds) and (B) in the late phase (6 minutes). Following PDT, ICGA reveals (C) an involuted polypoidal lesion with feeder vessel and BVN in the early phase (30 seconds) and (D) an absence of hypercyanescence in the late phase (10 minutes).

Abbreviations: ICGA, indocyanine green angiography; PDT, photodynamic therapy; BVN, branching vascular network.

location of the PCV corresponded to a study by Uyama et al on ICGA findings of Japanese patients with PCV, in which they proposed that PCV may be a peculiar form of CNV beneath the RPE and above the Bruch's membrane. ${ }^{8}$ Similar to this, histopathological examinations revealed that vessels found at the margins of type- 1 choroidal neovascular membranes tend to be matured and dilated, and therefore, PCV seemed to originate from longstanding type-1 (occult) CNV above Bruch's membrane and is not a primary choroidal vascular disorder. ${ }^{9}$ We postulated that a choroidal nevus may cause chronic inflammatory or degenerative changes overlying RPE, which may result in the growth of type- $1 \mathrm{CNV}$ and eventually lead to PCV lesions developing adjacent to the nevus. ${ }^{3}$

In our case, optical coherence tomography and angiography revealed a discrete orange grape-like lesion below the PED with early filling and leakage in the late phase associated with a BVN and an SRF, which were more typical of active PCV. Some may argue the current case to represent malignant transformation of choroidal nevus, since SRF was observed with an orange structure, which could be interpreted as pigment associated with choroidal melanoma. However, the pigmented lesion was flat, and the orange structure was adjacent to, not overlying, the lesion. Moreover, our case responded well to PDT being applied at the center of the PCV, not over the pigmented lesion, and has remained stable for 2 years post-PDT. These features support our case to be PCV secondary to choroidal nevus rather than malignant transformation.

Various treatments including thermal laser therapy have been used for PCV. ${ }^{10-15}$ ICGA-guided PDT was found to be effective in treating CNV associated with choroidal nevus; however, variable outcomes have been reported. ${ }^{11}$ PDT is also one of the most effective treatments for PCV, resulting in acuity improvement, leakage reduction, and complete PCV regression. ${ }^{13,14}$ However, a high recurrence rate and minimal BVN regression have been documented, with possible complications such as subretinal hemorrhage and RPE tears. ${ }^{16}$ The favorable outcome of PDT in our case was most likely due to the extrafoveal location of PCV and the patient's good initial acuity.

In PCV patients, vascular endothelial growth factor (VEGF) was found to be elevated in RPE and vascular endothelial cells. ${ }^{17}$ Intravitreal anti-VEGF injections for PCV have been shown to be effective in reducing SRF and improving vision; however, vascular abnormalities have often persisted. ${ }^{12,15}$ A previous study reported that combination treatment with PDT and anti-VEGF resulted in better acuity outcomes and a lower risk of developing PDT complications than photodynamic monotherapy. ${ }^{15}$ Lowering VEGF levels after PDT may be the key to preventing PCV recurrence and CNV development. However, Koh et al reported that in follow-up visits of up to 6 months, although PDT combined with ranibizumab was superior to ranibizumab monotherapy in achieving complete regression of PCV, no difference was found between the combination treatment and the photodynamic monotherapy. ${ }^{12}$

In this report, we present the case of a Caucasian female who developed symptomatic, active PCV at the edge of a stable choroidal nevus, which was successfully treated with one session of PDT, resulting in improved symptoms and fluid resolution, and no further treatment was required. ICGA is invaluable for diagnosing PCV and differentiating PCV from typical CNV, as PCV is a highly variable disorder. PCV secondary to choroidal nevus may be underdiagnosed. Further studies are required to understand the mechanisms of pathogenesis and evaluate optimal treatment options.

\section{Disclosure}

The authors report no conflicts of interest in this work. 


\section{References}

1. Singh AD, Kalyani P, Topham A. Estimating the risk of malignant transformation of a choroidal nevus. Ophthalmology. 2005;112(10):1784-1789.

2. Yannuzzi LA, Sorenson J, Spaide RF, Lipson B. Idiopathic polypoidal choroidal vasculopathy (IPCV). Retina. 1990;10(1):1-8.

3. Wong JG, Lai XJ, Sarafian RY, Wong HS, Smith JB. Polypoidal choroidal vasculopathy secondary to a stable choroidal nevus. Retin Cases Brief Rep. 2016;10(3):221-224.

4. Shields CL, Mashayekhi A, Materin MA, et al. Optical coherence tomography of choroidal nevus in 120 patients. Retina. 2005;25(3):243-252.

5. Callanan DG, Lewis ML, Byrne SF, Gass JD. Choroidal neovascularization associated with choroidal nevi. Arch Ophthalmol. 1993;111(6): 789-794.

6. Zografos L, Mantel I, Schalenbourg A. Subretinal choroidal neovascularization associated with choroidal naevus. Eur J Ophthalmol. 2004;14(2):123-131.

7. Papastefanou VP, Nogueira V, Hay G, et al. Choroidal naevi complicated by choroidal neovascular membrane and outer retinal tabulation. $\mathrm{BrJ}$ Ophthalmol. 2013;97(8):1014-1019.

8. Uyama M, Matsubara T, Fukushima I, et al. Idiopathic polypoidal choroidal vasculopathy in Japanese patients. Arch Ophthalmol. 1999;117(8): 1035-1042.

9. Freund KB. Different types of neovascularization that occur in AMD. The diagnosis and management of polypoidal choroidal vasculopathy (PCV). Presented at: The 8th Annual Retinal Physician Symposium; March 28-31, 2012; Miami Beach, FL.
10. Yuzawa M, Mori R, Haruyama M. A study of laser photocoagulation for polypoidal choroidal vasculopathy. Jpn J Ophthalmol. 2003;47(4): 379-384.

11. Parodi MB, Boscia F, Piermarocchi S, Ferrari TM, Furino C, Sborgia C. Variable outcome of photodynamic therapy for choroidal neovascularization associated with choroidal nevus. Retina. 2005;25(4):438-442.

12. Koh A, Lee WK, Chen LJ, et al. EVEREST study: efficacy and safety of verteporfin photodynamic therapy in combination with ranibizumab or alone versus ranibizumab monotherapy in patients with symptomatic macular polypoidal choroidal vasculopathy. Retina. 2012;32(8):1453-1464.

13. Otani A, Sasahara M, Yodoi Y, et al. Indocyanine green angiography: guided photodynamic therapy for polypoidal choroidal vasculopathy. Am J Ophthalmol. 2007;144(1):7-14.

14. Gomi F, Ohji M, Sayanagi K, et al. One-year outcomes of photodynamic therapy in age-related macular degeneration and polypoidal choroidal vasculopathy in Japanese patients. Ophthalmology. 2008;115(1):141-146.

15. Lai TY, Chan WM, Liu DT, Luk FO, Lam DS. Intravitreal bevacizumab (Avastin) with or without photodynamic therapy for the treatment of polypoidal choroidal vasculopathy. Br J Ophthalmol. 2008;92(5):661-666.

16. Kang HM, Kim YM, Koh HJ. Five-year follow-up results of photodynamic therapy for polypoidal choroidal vasculopathy. Am JOphthalmol. 2013;155(3):438-447.

17. Matsuoka M, Ogata N, Otsuji T, Nishimura T, Takahashi K, Matsumura M. Expression of pigment epithelium derived factor and vascular endothelial growth factor in choroidal neovascular membranes and polypoidal choroidal vasculopathy. Br J Ophthalmol. 2004;88(6):809-815.
International Medical Case Reports Journal

\section{Publish your work in this journal}

The International Medical Case Reports Journal is an international, peer-reviewed open-access journal publishing original case reports from all medical specialties. Previously unpublished medical posters are also accepted relating to any area of clinical or preclinical science. Submissions should not normally exceed 2,000 words or

\section{Dovepress}

4 published pages including figures, diagrams and references. The manuscript management system is completely online and includes a very quick and fair peer-review system, which is all easy to use. Visit http://www.dovepress.com/testimonials.php to read real quotes from published authors.

Submit your manuscript here: https://www.dovepress.com/international-medical-case-reports-journal-journal 